EFI-96-02

hep-th/9601179

\title{
Chern-Simons from Dirichlet 2-brane Instantons
}

\author{
Martin O'Loughlin \\ Enrico Fermi Institute \\ University of Chicago \\ 5640 S. Ellis Ave. \\ Chicago, IL 60637 USA \\ mjol@yukawa.uchicago.edu
}

\begin{abstract}
In the vicinity of points in Calabi-Yau moduli space where there are degenerating three-cycles the low energy effective action of type IIA string theory will contain significant contributions arising from membrane instantons that wrap around these three-cycles. We show that the world-volume description of these instantons is Chern-Simons theory.
\end{abstract}




\section{Introduction}

Recent progress has led to a deeper understanding of the conifold transition in the type IIB superstring theory [ [1]. The conifold is a point in Calabi-Yau moduli space where an $S^{3}$ degenerates. One can pass through the conifold to the other side through a small resolution by blowing up an $S^{2}$. In the mirror of this picture the conifold maps to a small resolution in IIA string theory. The image under mirror symmetry of the 3-branes wrapping the $S^{3}$ providing additional non-perurbative massless states is the 2-branes of the IIA theory wrapping the $S^{2}$ 's again giving additional massless states.

On the "other side" of the conifold in the type IIA theory we have a 3-cycle blowing up, however we have no 3-branes in IIA only even-branes. Therefore the important non-perturbative object to consider on this side of the conifold is the instanton formed by wrapping the Dirichlet membrane (D2-brane) world-volume of the IIA string theory [2] around the appropriate 3-cycle. We propose to study these D2-brane instantons by introducing their description via Chern-Simons theory. Other work on membrane instantons may be found in [3] though in that work the techniques used are significantly different to those we propose here. We believe that our methods will be complimentary to theirs and also to the other methods recently developed for the study of Dirichlet branes [4, 5, 6].

How do we describe strings attached to a Dirichlet 2-brane instanton that is wrapped around a 3-cycle of a Calabi-Yau manifold? It was shown by Polchinski [2] that the cycle around which the Dirichlet-brane is wrapped must be supersymmetric and also that the D-brane carries Ramond-Ramond charge. We will consider D2-brane instantons and will look for an open string field theory that describes the interaction between the strings and this supersymmetric field configuration on the boundary.

There is an open string theory that specifically fulfils the requirement that the string endpoints rest on a supersymmetric 3-cycle (actually a Lagrangian three-fold this being a necessary condition for the submanifold to be a supersymmetric 3-cycle) of the CalabiYau manifold. It was obtained by Witten [7] by topologically twisting the bosonic open string theory by an A-type twisting. One can show that this open string theory has a corresponding open string field theory that is simply Chern-Simons theory on the 3-cycle. We show that the instanton corrections to the effective field theory, in particular near points in moduli space where there are degenerating 3-cycles, may be calculated using Chern-Simons theory on the appropriate 3-cycle.

\section{The Details}

What are the properties of the three cycle around which we are wrapping the membrane? The three cycles that appear are those for which the wrapping of a D2-brane will 
not break more than half the supersymmetries of the string theory [8, 3,6]. This condition is the same as requiring that some global supersymmetry transformation restricted to the 3 -cycle can be undone by a $\kappa$-transformation. Let $i: M \rightarrow X$ be an embedding of a 3-cycle $M$ into a Calabi-Yau manifold $X$. In this case the condition of a supersymmetric three cycle is the requirement that $i^{*} \Omega \sim V$ and $i^{*} \omega=0$, where $V$ is the volume of the three cycle, $\Omega$ is the holomorphic three form on $X$ and $\omega$ is the Kahler form on $X$. Such cycles minimize the p-brane world-volume [3]. For a 3-cycle to be a Lagrangian submanifold we need $i^{*} \omega=0$ and thus supersymmetric 3-cycles are three-dimensional Lagrangian submanifolds.

For the A-twisted bosonic string with Dirichlet boundary conditions in M, BRS invariance of the boundary conditions is fulfilled if the three manifold described by the boundary conditions is a Lagrangian submanifold with a flat connection living on it. This was explained by Witten in [7] and we will explain it in some detail below. An earlier construction where the 3-manifold is found as the fixed set of an involution is described in [9] in which case the 3-manifold was a Lagrangian submanifold by construction. Thus we have discovered that the A-twisted open string is the appropriate description for strings in the presence of D2-brane instantons wrapped around a supersymmetric 3-cycle. As discussed in [10] the topologically twisted string theory calculates certain quantities in the supersymmetric string theories. For the A-type twisting corrections to the effective action of the type IIA superstring are obtained.

We will now follow the discussion of Witten in [7] and describe in detail the boundary theory of the A-twisted string theory with a Calabi-Yau target space. As above, let $X$ be the Calabi-Yau target space and $\Phi: \Sigma \rightarrow X$ the map from the Riemann surface into the target space. In the A-twisted string theory we have the three fields $\phi$ (the local components of $\Phi), \chi$ and $\psi$ with the following fermionic symmetry $(I, J$ are co-ordinate indices on $X, x^{\alpha}, z, \bar{z}$ co-ordinate indices on $\Sigma$ and $i, \bar{i}$ spinor indices on $X$ ),

$$
\begin{aligned}
\delta \phi^{I} & =i \alpha \chi^{I} \\
\delta \chi^{I} & =0 \\
\delta \psi_{z}^{\bar{i}} & =-\alpha \partial_{z} \phi^{\bar{i}}-i \alpha \chi^{\bar{j}} \Gamma_{\bar{j} \bar{m}}^{\bar{i}} \psi_{z}^{\bar{m}} \\
\delta \psi_{\bar{z}}^{i} & =-\alpha \partial_{\bar{z}} \phi^{i}-i \alpha \chi^{j} \Gamma_{j m}^{i} \psi_{\bar{z}}^{m}
\end{aligned}
$$

The boundary condition is $\partial \Sigma \rightarrow M$ where $M$ is required to be a Lagrangian submanifold. We define $Q$ as the generator of these transformations; $\delta \Lambda=-i \alpha\{Q, \Lambda\}$. Our Lagrangian is given by $L=i\{Q, V\}$ and $T_{\alpha \beta}=i\left\{Q, b_{\alpha \beta}\right\}$, where $V=t \int_{\Sigma} d^{2} z g_{i \bar{j}}\left(\psi_{z}^{\bar{i}} \partial_{\bar{z}} \phi^{j}+\partial_{z} \phi^{\bar{i}} \psi_{\bar{z}}^{j}\right)$ and $b_{\alpha \beta}=i t g_{I J}\left(\psi_{\alpha}^{I} \partial_{\beta} \phi^{J}+\psi_{\beta}^{I} \partial_{\alpha} \phi^{J}-h_{\alpha \beta} h^{\sigma \tau} \psi_{\sigma}^{I} \partial_{\tau} \phi^{J}\right)$. 
In the 3-manifold $\mathrm{M}$ there is a gauge field coming from the Chan-Paton factors of the open string theory. Denote this field by $A$ and its field strength by $F$. From the recent study of p-brane bound states [4] we expect an n-wrapped D2-brane to have associated with it an open string theory with a $U(n)$ Chan-Paton factor. This non-abelian group arises from the $\mathrm{n}$ different leaves of the multiply wrapped 2-brane to which the open string may attach. For the soliton world volume theory formed by pulling back the target space effective action to the world-volume of a Dn-brane wrapped about an n-cycle [4] the multiply wrapped configuration was not allowed because it was argued to not produce the correct counting of BPS states and to have an inconsistent world-volume field theory. For instantons we claim that to be saddle points the membranes must wrap Euclidean supersymmetric 3-cycles and as stability is not an issue for instantons, multiple wrappings of the membrane world-volume around the supersymmetric 3-cycle are allowed.

The boundary term in this twisted open string theory is a Wilson line running around the boundary of $\Sigma$ and the variation of the boundary term under the fermionic symmetry is

$$
\delta\left(\operatorname{Tr} P \exp \oint_{\partial \Sigma} \Phi^{*}(A)\right)=\operatorname{Tr}\left(\oint_{\partial \Sigma} d \tau i \alpha \chi^{I} \frac{d \phi^{J}}{d \sigma} F_{I J}(\tau) P \exp \oint_{\partial \Sigma ; \tau} \Phi^{*}(A)\right)
$$

and $\oint_{\partial \Sigma ; \tau}$ means the integral around $\partial \Sigma$ starting and ending at $\tau$. The BRS invariance of this boundary condition shows that the world-sheet theory can be consistently coupled to flat connections on the D-instanton world-volume.

We will describe the corresponding string field theory. To do this we first look at the eigenfunctions of $L_{0}$,

$$
L_{0}=\frac{1}{2} \int_{0}^{\pi}\left(-\frac{1}{t} g^{I J} \frac{\delta^{2}}{\delta \phi^{I} \delta \phi^{J}}+t g_{I J} \frac{d \phi^{I}}{d \sigma} \frac{d \phi^{J}}{d \sigma}+\ldots\right) .
$$

For weak coupling $(t \rightarrow \infty)$ where the theory is exact $L_{0}=0$ requires that $\frac{d \phi^{I}}{d \sigma}=0$ and the entire world-sheet is mapped into the 3-cycle. We can then write the string field functional in the three-cycle as, $\mathcal{A}=c(q)+\chi^{a} A_{a}(q)+\chi^{a} \chi^{b} B_{a b}(q)+\ldots$ where $q$ is a local co-ordinate on the three manifold $M$. With the Chan-Paton factors for the case where we have the D2-brane wrapped multiple times around the three-cycle $A_{a}$ is a non-abelian gauge field on $M$. Also $\{Q, \phi\}=-\chi$ and $\{Q, \chi\}=0$ which are solved on $M$ by $Q=d$ and $\chi=-d \phi$. With these relations plugged into the open string field theory action the open string world lines in $M$ look just like the fattened matrix propagators of the matrix field $A$ in $M$,

$$
\begin{aligned}
S_{\text {osft }} & =\frac{k}{2} \int\left(\mathcal{A} \star Q \mathcal{A}+\frac{2}{3} \mathcal{A} \star \mathcal{A} \star \mathcal{A}\right) \\
& =\frac{k}{2} \int_{M}\left(A \wedge d A+\frac{2}{3} A \wedge A \wedge A\right)
\end{aligned}
$$


The total membrane contribution will then involve the Chern-Simons theory plus the membrane self-energy coming from the $2+1$ supermembrane action, analytically continued to the Euclidean metric,

$$
S_{m e m}=\frac{T_{m e m}}{2} \int d^{3} \sigma \sqrt{\gamma}\left(\gamma_{i j} \partial_{m} X^{i} \partial_{n} X^{j}-\frac{1}{2}+\gamma^{i j} \gamma^{k l} F_{i k} F_{j l}\right)+\ldots
$$

Wrapping the membrane world-volume around the Lagrangian three-cycle sets $F=0$ (from (2.2) or (2.4) ) and thus the membrane self-energy weighting is the action of the three-cycle world-volume. For a generic point in the moduli space of the Calabi-Yau manifold, we may expect that the world-volume instantons are not dominant in the partition function due to the weight of the world-volume coming from (2.5). By the Lagrangian submanifold condition the three-cycle has minimal volume and the instanton corrections can therefore become significant near points in the moduli space where a three-cycle is degenerate. We claim that these corrections can be obtained from the Chern-Simons theory described above, with an additional contribution coming from the supermembrane world-volume theory.

Putting all these pieces together and using the dilute instanton gas approximation to carry out the instanton summation, the total expression for the D2-brane instanton correction to the partition function when the instanton is obtained by wrapping a 3 -sphere is

$$
Z_{\text {inst }}=\sum_{m=1}^{\infty}(K V)^{m} \sum_{\left\{n_{i}\right\}_{1}^{m}} \prod_{j=1}^{m} e^{-n_{j} S_{m e m}} Z\left[U\left(n_{j}\right), k ; S^{3}\right] \sqrt{n_{j} S_{m e m}}
$$

where

$$
Z\left[U(n), k ; S^{3}\right]=(k+n)^{-n / 2} \sqrt{\frac{(k+n)}{n}} \prod_{j=1}^{n-1}\left(2 \sin \left(\frac{j \pi}{n+k}\right)\right)^{n-j},
$$

$V$ is the volume of the space-time in which the instantons live, $\mathrm{K}$ is a factor that arises from the closed string fluctutations around the instanton configuration and $S_{m e m}$ is evaluated for a D2-brane wrapping the minimal 3-cycle of a Calabi-Yau manifold (we are considering for simplicity the case where we have $h_{2,1}=1$ ). We assert that $K$ is a constant independent of the wrapping number. In field theory $K$ comes from the correction to the flat space field fluctuations caused by the presence of the localized instanton configurations. As the instanton size is fixed by the string gas that surrounds it, and as this is not strongly dependent upon the number of times the membrane wraps the 3-cycle, we expect $K$ to be constant. The Chern-Simons coupling constant $1 / \sqrt{k}$ is equal to the open string coupling constant which is in turn equal to the square root of the closed string coupling. The Dbrane tension $T_{m e m}$ which appears in the membrane action (2.5) is proportional to the disk 
amplitude of the string theory (with the disk boundary resting on the Ramond-Ramond charged D-brane) as argued by Polchinski [11], which is in turn proportional to $1 / g_{s t}$. So the $e^{-n S_{m e m}}$ weighting factor with $S_{m e m} \sim \frac{1}{g_{s t}} V_{3-c y c l e}$ is the source of the $e^{-1 / g_{s t}}$ corrections argued to be present in string theory by Shenker [12]. This will contribute significantly to the partition function when the volume of the compactification 3-cycle is small, for example for the type IIA string near the conifold point in the moduli space of the quintic threefold.

The instantons that arise bear a passing resemblance to the instantons of the Fermi sea picture of the $c=1$ string theory. This similarity is somewhat strengthened by recent conjectures relating the $c=1$ model at the self-dual radius to the string physics of the conifold transition. The instantons of $c=1$ arise when one continues the Fermi sea to Euclidean space; $u v=\mu$ becomes $x^{2}+y^{2}=\mu$. The instanton is a single fermion that runs around the circular Fermi sea of this Euclidean continuation. For the conifold in the IIA theory we have a 3 -sphere $\sum_{i=0}^{3} x_{i}^{2}=\mu$ about which our membrane instanton is wrapped, and this 3-sphere is conjectured [13] to be in a direct relationship to the Fermi sea of $c=1$ and also to the ground ring [14] of $c=1$. This implies an identification between $k$ and $\mu$. With this in hand the conifold is approached by taking $\mu \rightarrow 0$ and thus $k \rightarrow 0$. In this limit the partition function (2.7) simplifies somewhat when $n$ is also large [15] and the ubiquitous Bernoulli numbers arise, giving hope that some closed form expression may be obtained国.

The dilute instanton gas approximation that we have used to derive the expression for $Z_{\text {inst }}$ should be very good. The main requirement for the absence of instanton interactions is that the size of the instantons be "small". In our case the instanton is wrapped around a cycle of the internal Calabi-Yau manifold and so is essentially pointlike from the point of view of the four-dimensional physics. Even taking account of the strings attached to the instanton its size will be no greater than the string scale and thus one would expect the dilute instanton gas approximation to be excellent.

\section{Further Directions}

For more general degenerations of the type discussed in the recent literature one needs to consider in addition to the open strings attached to a single Dirichlet instanton the

1 A relationship between Chern-Simons theory, the conifold and the $c=1$ string at the selfdual radius was also observed recently in [16]. This proposal is distinct from ours in that ours involves the "other side" of the conifold. 
strings with different ends attached to different instantons. Obviously when the individual D-instantons are widely separated such contributions will be supressed by the world-volume of the stretched string. Close to enhanced symmetry points and moduli space singularities these contributions will be important. In addition to the weighting factor that comes from the supermembrane world-volume theory, we must allow the string field theory that lives on the instanton world-volume to include diagrams where only one end of the string rests in a particular manifold. Thus we need to add to the Chern-Simons theory some particles living in the fundamental representation of the gauge group. We also need to consider wrapping the membranes around non-trivial 3-cycles with topology other than the $S^{3}$ considered here. In [5] some of the 3-cycles have the topology of $S^{2} \times S^{1}$. The partition function of (2.7) is then modified [17]. According to the discussion of [7] in situations where the Lagrangian 3-fold has non-trivial topology, as for $S^{2} \times S^{1}$, additional topological terms can be added to the string field theory action. The significance of such additional terms needs to be understood in this context.

In the spirit of using field theory on world-volumes to discuss string theory interacting with D-branes [4,5,6, 18] we may try to extend the Chern-Simons theory for instance by coupling it to some fermions living on the world-volume. With additional matter present we have the possibility of phase transitions and we may inquire as to whether these have some interpretation in terms of D-brane bound states or collective excitations of D-branes.

Within the Chern-Simons theory we can also calculate the contributions of these fourdimensional instanton (point events in the four-dimensional space-time) configurations to various coupling constants of the low energy effective action (for example the calculation of the instanton correction to the four-fermi interaction in the low energy effective theory of IIA strings in four dimensions in [3]). Such instanton corrections to string scattering should be found by calculating Wilson loop correlators in the Chern-Simons theory [17]. In more generality we could investigate the meaning of the Wilson loop correlators that one calculates in the Chern-Simons theory. The skein relations of the Chern-Simons theory allow us to unknot configurations of Wilson loops introducing Möbius band configurations. For the two loop correlators this seems to indicate that the linked loops calculate the full answer and the unlinked loops calculate the correction excluding the unoriented worldsheets attached to the D2-brane instanton. Certainly the partition sum includes these unoriented configurations.

Finally we need a means to investigate the small structure of the type conjectured recently in [19] or as observed within the context of Calabi-Yau moduli space in [20]. Our instantons wrapped about the collapsing three-cycles will become -1-branes in the limit of zero volume. Such configurations are conjectured in [19] to give rise to point-like structure 
in string theory. By appropriate manipulation of the Chern-Simons theory we may develop some tools to investigate this conjecture. Further development of the relationship between the D2-brane instantons and the eigenvalue instantons of the $c=1$ matrix model is also desirable. There is certainly much more to be done than has been done.

\section{Acknowledgements}

I would like to thank Jeff Harvey, Albion Lawrence and especially Emil Martinec for discussions. This work was supported by DOE grant DE-FG02-90ER40560. 


\section{References}

[1] A. Strominger, "Massless Black Holes and Conifolds in String Theory", Nucl. Phys. B451 (1995), 96, hep-th/9504090. B. Greene, D. Morrison and A. Strominger, "Black Hole Condensation and the Unification of String Vacua", Nucl. Phys. 451 (1995), 109, hep-th/9504145.

[2] J. Polchinski, "Dirichlet Branes and Ramond-Ramond Charges" Phys. Rev. Lett. 75 (1995), 4724, hep-th/9510017.

[3] K. Becker, M. Becker and A. Strominger, "Fivebranes, Membranes and Nonperturbative String Theory", Nucl. Phys. B456 (1995), 130, hep-th/9507158.

[4] E. Witten, "Bound States of Strings and p-branes", hep-th/9510135

[5] M. Bershadsky, V. Sadov and C. Vafa, "D Strings on D Manifolds", hep-th/9510225.

[6] M. Bershadsky, V. Sadov and C. Vafa, "D-branes and Topological Field Theories", hep-th/9511222.

[7] E. Witten, "Open Strings as Chern-Simons", hep-th/9207094.

[8] J. Hughes, J. Liu and J. Polchinski, "Supermembranes", Phys. Lett. B180 (1986), 370.

[9] P. Hořava, "Equivariant Topological Sigma Models", Nucl. Phys. B418 (1994), 571, originally PRA-HEP-90/18, hep-th/9309124.

[10] M. Bershadsky, S. Cecotti, H. Ooguri and C. Vafa, "Kodaira-Spencer Theory of Gravity and Exact Results for Quantum String Amplitudes", Comm. Math. Phys. 165 (1994), 311, hep-th/9309140

[11] J. Polchinski, "Combinatorics of Boundaries in String Theory", Phys. Rev. D50 (1994), 6041, hep-th/9407031.

[12] S. Shenker, "The Strength of Nonperturbative Effects in String Theory", In Cargese 1990, Proceedings, Random surfaces and quantum gravity, 191.

[13] D. Ghoshal and C. Vafa, " $c=1$ String as the Topological Theory of the Conifold", Nucl. Phys. B453 (1995), 121, hep-th/9506122.

[14] E. Witten, "Ground Ring of Two-dimensional String Theory", Nucl. Phys. B373 (1992), 187, hep-th/9108004.

[15] V. Periwal, "Topological Closed String Interpretation of Chern-Simons Theory", Phys. Rev. Lett. 71 (1993), 1295, hep-th/9305115.

[16] D. Jatkar and B. Peeters, "String Theory near a Conifold singularity", Phys. Lett. B362 (1995), 73, hep-th/9508044.

[17] E. Witten, "Quantum Field Theory and the Jones Polynomial", Comm. Math. Phys. 121 (1989), 351.

[18] M. Douglas, "Branes within Branes", hep-th/9512077.

[19] S. Shenker, "Another Length Scale in String Theory?", hep-th/9509132.

[20] P. Aspinwall, B. Greene and D. Morrison, "Measuring Small Distances in $N=2$ sigma models", Nucl. Phys. B420 (1994), 184, hep-th/9311042. 